\title{
36. DATA REPORT: STABLE ISOTOPIC STRATIGRAPHY OF THE PALEOGENE PELAGIC CAP AT SITE 865, ALLISON GUYOT ${ }^{1}$
}

\author{
Timothy J. Bralower, ${ }^{2}$ Matthew Parrow, ${ }^{2}$ Ellen Thomas, ${ }^{3}$ and James C. Zachos ${ }^{4}$
}

\begin{abstract}
Intensive stable isotopic investigations were conducted on the upper Paleocene to upper Eocene pelagic cap sequence recovered at Site 865, Allison Guyot, Mid-Pacific Mountains. The sequence consists of calcareous ooze with an unusually high content of well-preserved planktonic foraminifers. Benthic foraminifers, although exceptionally rare, are also nearly unaltered. Isotopic analyses were performed on three separate planktonic (species of Acarinina, Morozovella, and Subbotina) and four benthic taxa (species of Cibicidoides and Lenticulina, Gavelinella beccariiformis, and Nuttallides truempyi). We present results of detailed stable isotopic investigations $\left(\delta^{18} \mathrm{O}\right.$ and $\left.\delta^{13} \mathrm{C}\right)$ of the uppermost Paleocene and lowermost Eocene intervals in two holes $(865 \mathrm{~B}$ and $865 \mathrm{C})$ and of the entire upper Paleocene to upper Eocene in Hole 865B.
\end{abstract}

\section{INTRODUCTION}

Site $865\left(18^{\circ} 26^{\prime} \mathrm{N}, 179^{\circ} 33^{\prime} \mathrm{W}\right)$ is situated at a water depth of 1530 $\mathrm{m}$ atop Allison Guyot in the Mid-Pacific Mountains. A relatively expanded and largely complete upper Paleocene to lower Oligocene sequence was recovered in the pelagic cap part of this site (Bralower and Mutterlose, this volume). Sediments, largely calcareous oozes, contain a sandy texture as a result of the high content of planktonic foraminifers. These microfossils are predominantly of exceptional preservation and therefore are suitable for isotopic investigations. In this report, we document the results of 586 stable isotopic measurements of planktonic and benthic foraminifers from the Paleogene section of Site 865. Detailed discussion and interpretation of these data are presented in Bralower et al. (unpubl. data) and Zachos et al. (unpubl. data). Benthic foraminiferal assemblages are discussed in detail by Thomas (unpubl. data).

\section{METHODS AND PROCEDURES}

One to three samples per core section were selected for isotopic measurement from Hole 865B, with the densest sampling in the upper Paleocene to lower Eocene interval. A more detailed sample set (one sample every $10 \mathrm{~cm}$ ) was taken close to the benthic foraminiferal extinction horizon in the upper Paleocene in both Holes $865 \mathrm{~B}$ and $865 \mathrm{C}$. One $20-\mathrm{cm}^{3}$ sample was taken per section in Hole $865 \mathrm{~B}$ for benthic foraminiferal analysis. All other samples ranged in volume between 5 and $10 \mathrm{~cm}^{3}$. All samples were split, dried, and washed with water having a pH of 8 through sieves having screen openings of 250 , 125,63 and $38 \mu \mathrm{m}$. The $<38-\mu \mathrm{m}$ fraction was passed through a millipore filter and saved for future fine-fraction isotopic investigations. All other fractions were oven-dried at $60^{\circ} \mathrm{C}$. The $>250-\mu \mathrm{m}$ fraction was dry-split into various size fractions: $>400,355-400$, $300-355$, and $250-300 \mu \mathrm{m}$. To reduce ontogenetic and vital effects on the interpretation of stable isotope results (e.g., Shackleton et al., 1985a; Corfield and Cartlidge, 1991; Pearson et al., 1993), almost all of our planktonic foraminiferal isotopic measurements were performed on the $300-355 \mu \mathrm{m}$ fraction. Different size fractions were

\footnotetext{
'Winterer, E.L., Sager, W.W., Firth, J.V., and Sinton, J.M. (Eds.), 1995. Proc. ODP, Sci. Results, 143: College Station, TX (Ocean Drilling Program).

${ }^{2}$ Department of Geology, University of North Carolina, Chapel Hill, NC 27599-3315, U.S.A

${ }^{3}$ Department of Geology and Geophysics, Yale University, New Haven, CT 06511 , U.S.A.

${ }_{4}^{4}$ Earth Science Board, University of California, Santa Cruz, CA 95064, U.S.A.
}

used only when the taxon of interest was not observed in large enough quantities in the $300-355 \mu \mathrm{m}$ fraction

Various taxa have been used for Paleogene planktonic foraminifer isotope stratigraphy (e.g., Shackleton et al., 1984; Boersma et al., 1987; Stott et al., 1990; Zachos et al., 1994). Species of Morozovella and Acarinina were chosen for analysis on the basis of (1) taxonomic distinctiveness and (2) overlapping stratigraphic ranges that span the interval of interest. Based on these criteria, the following species were selected: Morozovella velascoensis (upper Paleocene), Morozovella subbotinae (uppermost Paleocene-lower Eocene), Morozovella aragonensis (lower to middle Eocene), and Morozovella lehneri (middle-upper Eocene); Acarinina mckannai (upper Paleocene), Acarinina soldadoensis (upper Paleocene-lower Eocene), and Acarinina bullbrooki (lower-upper Eocene). As is common practice, we have combined species of Subbotina because of taxonomic uncertainties. Selection of planktonic foraminifers for isotopic measurement was performed at UNC-CH largely by Parrow and Bralower. Samples of each taxon were verified by I. Premoli Silva, W. Sliter, and D.C. Kelly. Initially, 25 specimens of each species were chosen; however, the eight to 10 best-preserved specimens were selected for isotopic measurement.

Samples for benthic foraminiferal studies were first dried in an oven at $60^{\circ} \mathrm{C}$, then soaked and gently shaken in distilled water overnight. If samples had not disaggregated the next day, detergent was added and the samples were again gently shaken overnight. All samples disaggregated after this treatment. Samples were then washed through a $63-\mu \mathrm{m}$ sieve, and the coarse fraction was dried in an oven at $60^{\circ} \mathrm{C}$, and weighed. The whole fraction larger than $63 \mu \mathrm{m}$ was used, because of the importance of small specimens for paleoecological studies, and because we want to compare results with those of Thomas (1990). Preservation of benthic foraminifers is very good in most samples, although some samples contained yellowed, corroded specimens that were probably reworked from older materials. These specimens were not used for isotopic analysis. Benthic foraminiferal specimens are rare in all samples studied, with numbers varying from about 200 to $3000 / \mathrm{g}$; this is about 10 to 100 times fewer specimens per gram than in samples of equivalent age and paleodepth from Maud Rise (Weddell Sea, Antarctica; Thomas, 1990). As a result, numerous samples had insufficient specimens of particular taxa for isotopic analysis. However, we have been able to isolate between five and 20 specimens of select taxa in a number of samples. We have selected Nuttallides truempyi and species of Cibicidoides throughout the investigated section, and Gavelinella beccariiformis and species of Lenticulina in samples from the uppermost Paleocene. All processing and selection of benthic foraminifers was performed by Thomas. 
Isotopic analyses were performed in the Stable Isotope laboratory at the University of Michigan. Individual foraminifers were sonicated in distilled water to remove adhering particles and roasted in vacuo at $380^{\circ} \mathrm{C}$. The specimens $(8-10$ individuals of planktonic foraminifers, 4-10 specimens of benthic foraminifers) were processed in a CarboKiel Mat 251 carbonate digestion device. Each sample was reacted in an individual vessel with three drops of phosphoric acid at $75^{\circ} \mathrm{C}$. The resulting $\mathrm{CO}_{2}$ was isolated in a single-step distillation and then introduced directly to the MAT-251 mass-spectrometer for measurement. NBS-18, -19, and -20, as well as an in-house standard LV-2, were measured on a daily basis to monitor instrument calibration and analytical accuracy. All values are expressed in the $(\delta)$ notation where,

$$
\delta(\%)=\frac{{ }^{13} \mathrm{C} /{ }^{12} \mathrm{C}(\text { samp })-{ }^{13} \mathrm{C} /{ }^{12} \mathrm{C}(\mathrm{std})}{{ }^{13} \mathrm{C} /{ }^{12} \mathrm{C}(\mathrm{std})} \times 10^{3}
$$

relative to the PDB standard. Average precision determined from 40 replicate analyses of planktonic foraminifers was better than $\pm 0.1 \%$ for both oxygen and carbon compositions, but significantly lower than this for benthic foraminifers. Because of the significant length of time (nine months) over which analyses were made, in the third run we conducted 10 replicate analyses from the first two runs to assess possible machine "drift." Based on the results of these replicates, we determined that, on average, replicates were $0.2 \%$ heavier in the third run for both $\delta^{18} \mathrm{O}$ and $\delta^{13} \mathrm{C}$, and thus these values have been corrected accordingly.

\section{RESULTS}

Data have been compiled in Tables 1 and 2. We list, along with the sample, depth, taxon measured, and stable isotopic data, the size fraction and run number. In Table 1, we include data from Hole 865B in which we have measured samples from the entire upper Paleocene to upper Eocene interval. In Table 2, we compile data from Hole $865 \mathrm{C}$ that has been sampled in great detail in the interval close to the Paleocene/Eocene boundary. Interpretation of the data reported here is given in Bralower et al. (unpubl. data) and Zachos et al. (unpubl. data).

\section{ACKNOWLEDGMENTS}

We thank K.C. Lohmann for the use of the Michigan Stable Isotope Laboratory and for running analyses for us. We gratefully acknowl- edge discussions with Steve D'Hondt, D. Clay Kelly, Isabella Premoli Silva, and William Sliter on taxonomic concepts. Research supported by JOI-USSAC.

\section{REFERENCES*}

Boersma, A., Premoli Silva, I., and Shackleton, N.J., 1987. Atlantic Eocene planktonic foraminiferal paleohydrographic indicators and stable isotope paleoceanography. Paleoceanography, 2:287-331.

Corfield, R.M., and Cartlidge, J.E., 1991. Isotopic evidence for the depth stratification of fossil and Recent Globigerinina: a review. Hist. Biol., 5:37-63.

Pearson, P.N., Shackleton, N.J., and Hall, M.A., 1993. Stable isotopic paleoecology of middle Eocene planktonic foraminifera and multi-species isotope stratigraphy, DSDP Site 523, South Atlantic. J. Foraminiferal Res., 23:123-140.

Shackleton, N.J., Corfield, R.M., and Hall, M.A., 1985. Stable isotope data and the ontogeny of Paleocene planktonic foraminifera. J. Foraminiferal Res., 15:321-336.

Shackleton, N.J., Hall, M.A., and Boersma, A., 1984. Oxygen and carbon isotope data from Leg 74 foraminifers. In Moore, T.C., Jr., Rabinowitz, P.D., et al., Init. Repts. DSDP, 74: Washington (U.S. Govt. Printing Office), 599-644.

Stott, L.D., Kennett, J.P., Shackleton, N.J., and Corfield, R.M., 1990. The evolution of Antarctic surface waters during the Paleogene: inferences from the stable isotopic composition of planktonic foraminifers, ODP Leg 113. In Barker, P.F., Kennett, J.P., et al,, Proc. ODP, Sci. Results, 113: College Station, TX (Ocean Drilling Program), 849-863.

Thomas, E., 1990. Late Cretaceous through Neogene deep-sea benthic foraminifers (Maud Rise, Weddell Sea, Antarctica). In Barker, P.F., Kennett, J.P., et al., Proc. ODP, Sci. Results, 113: College Station, TX (Ocean Drilling Program), 571-594.

Zachos, J.C., Stott, L.D., and Lohmann, K.C., 1994. Evolution of early Cenozoic marine temperatures. Paleoceanography, 9:353-387.

Abbreviations for names of organizations and publications in ODP reference lists follow the style given in Chemical Abstracts Service Index (published by American Chemical Society).

Date of initial receipt: 1 December 1993

Date of acceptance: 25 May 1994

Ms 143SR-215 
Table 1. Stable isotopic data for Hole 865B.

\begin{tabular}{|c|c|c|c|c|c|c|c|c|c|c|}
\hline $\begin{array}{l}\text { Core, section, } \\
\text { interval }(\mathrm{cm})\end{array}$ & $\begin{array}{l}\text { Depth } \\
\text { (mbsf) }\end{array}$ & Taxon & $\begin{array}{l}\text { Size } \\
(\mu \mathrm{m})\end{array}$ & $\begin{array}{l}\delta^{13} \mathrm{C} \\
(\%)\end{array}$ & $\begin{array}{l}\delta^{18} \mathrm{O} \\
(\% \infty)\end{array}$ & Run & $\begin{array}{l}\text { Core, section, } \\
\text { interval }(\mathrm{cm})\end{array}$ & $\begin{array}{l}\text { Depth } \\
\text { (mbsf) }\end{array}$ & Taxon & $\begin{array}{l}\text { Size } \\
(\mu \mathrm{m})\end{array}$ \\
\hline Benthic foraminifer & & & & & & & $7 \mathrm{H}-1,118-120$ & 57.18 & M. lehneri & $300-355$ \\
\hline $3 \mathrm{H}-2,120-125$ & 20.70 & Cibicidoides sp. & $>125$ & 0.92 & 1.03 & 3 & $6 \mathrm{H}-4,70-72$ & 51.70 & M. aragonensis & $300-355$ \\
\hline $3 \mathrm{H}-3,120-125$ & 22.20 & Cibicidoides sp. & $>125$ & 0.77 & 0.62 & 3 & $6 \mathrm{H}-5,70-72$ & 53.20 & M. aragonensis & $300-355$ \\
\hline $3 \mathrm{H}-4,120-125$ & 23.70 & Cibicidoides sp. & $>125$ & 0.88 & 1.01 & 3 & $7 \mathrm{H}-1,120-125$ & 57.20 & M. aragonensis & $300-355$ \\
\hline $3 \mathrm{H}-5,120-125$ & 25.20 & Cibicidoides sp. & $>125$ & 0.46 & 0.91 & 3 & $7 \mathrm{H}-4,68-70$ & 61.18 & M. aragonensis & $300-355$ \\
\hline $3 \mathrm{H}-6,120-125$ & 26.70 & Cibicidoides sp. & $>125$ & 0.39 & 0.85 & 3 & $8 \mathrm{H}-1,89-91$ & 66.39 & M. aragonensis & $300-355$ \\
\hline $\begin{array}{l}4 \mathrm{H}-4,120-125 \\
5 \mathrm{H}-2,120-125\end{array}$ & $\begin{array}{l}33.20 \\
39.70\end{array}$ & $\begin{array}{l}\text { Cib. prae. } \\
\text { Cibicidoides sp. }\end{array}$ & $\begin{array}{l}>125 \\
>125\end{array}$ & $\begin{array}{l}0.70 \\
0.50\end{array}$ & $\begin{array}{l}0.36 \\
0.68\end{array}$ & $\begin{array}{l}3 \\
3\end{array}$ & $8 \mathrm{H}-2,70-72$ & 67.70 & M. aragonensis & $300-355$ \\
\hline $6 \mathrm{H}-2,120-125$ & 49.20 & Cibicidoides sp. & $>125$ & 0.51 & $\begin{array}{l}0.08 \\
0.49\end{array}$ & $\begin{array}{l}3 \\
3\end{array}$ & $8 \mathrm{H}-3,70-72$ & 69.20 & $M$. aragonensis & $300-355$ \\
\hline $6 \mathrm{H}-4,120-125$ & 52.20 & Cib. prae. & $>125$ & 0.61 & 0.52 & 3 & $8 \mathrm{H}-4,67-69$ & 70.67 & M. aragonensis & $300-355$ \\
\hline $6 \mathrm{H}-6,111-116$ & 55.11 & Cibicidoides sp. & $>125$ & 0.38 & 0.33 & 3 & $8 \mathrm{H}-5,70-72$ & 72.20 & $M$. aragonensis & $300-355$ \\
\hline $8 \mathrm{H}-2,120-125$ & 68.20 & Cibicidoides sp. & $>125$ & 0.52 & 0.46 & 3 & $8 \mathrm{H}-6,70-72$ & 73.70 & M. aragonensis & $300-355$ \\
\hline $8 \mathrm{H}-6,106-111$ & 74.06 & Cibicidoides sp. & $>125$ & 0.49 & 0.00 & 3 & $9 \mathrm{H}-1,20-22$ & 75.20 & M. aragonensis & $300-355$ \\
\hline $9 \mathrm{H}-2,120-125$ & 77.70 & Cibicidoides sp. & $>125$ & 1.10 & -0.35 & 3 & $9 \mathrm{H}-1,70-72$ & 75.70 & M. aragonensis & $300-355$ \\
\hline $9 \mathrm{H}-4,120-125$ & 80.70 & Cibicidoides sp. & $>125$ & 0.78 & -0.12 & 3 & $9 \mathrm{H}-1,120-125$ & 76.20 & M. aragonensis & $300-355$ \\
\hline $10 \mathrm{H}-2,120-125$ & 87.70 & Cib. prae. & $>125$ & 0.03 & -0.53 & 3 & $9 \mathrm{H}-2,20-22$ & 76.70 & M. aragonensis & $300-355$ \\
\hline $10 \mathrm{H}-5,111-116$ & 91.61 & Cibicidoides sp. & $>125$ & 0.49 & -0.40 & 1 & $9 \mathrm{H}-2,70-72$ & 77.20 & M. aragonensis & $300-355$ \\
\hline $11 \mathrm{H}-2,120-125$ & 96.70 & Cibicidoides sp. & $>125$ & 0.63 & -0.10 & 3 & $9 \mathrm{H}-2,120-125$ & 77.70 & M. aragonensis & $300-355$ \\
\hline $11 \mathrm{H}-4,120-125$ & 99.70 & Cib.prae & $>125$ & 0.85 & -0.26 & 3 & $9 \mathrm{H}-3,20-22$ & 78.20 & M. aragonensis & $300-355$ \\
\hline $11 \mathrm{H}-6,47-49$ & 101.97 & Cibicidoides sp. & $>125$ & 1.01 & -0.29 & 3 & $9 \mathrm{H}-3,70-72$ & 78.70 & M. aragonensis & $300-355$ \\
\hline $11 \mathrm{H}-\mathrm{CC}, 9-12$ & 102.53 & Cibicidoides sp. & $>125$ & 0.89 & -0.04 & 3 & $9 \mathrm{H}-3,120-125$ & 79.20 & $M$, aragonensis & $300-355$ \\
\hline $12 \mathrm{H}-1,10-12$ & 103.60 & Cibicidoides sp. & $>125$ & 0.04 & -0.58 & 1 & $9 \mathrm{H}-4,10-12$ & 79.60 & M. aragonensis & $300-355$ \\
\hline $12 \mathrm{H}-1,10-12 \mathrm{D}$ & 103.60 & Cibicidoides sp. & $>125$ & -0.12 & -0.21 & 1 & $9 \mathrm{H}-4,70-72$ & 80.20 & M. aragonensis & $300-355$ \\
\hline $12 \mathrm{H}-1,40-42$ & 103.90 & Cibicidoides sp. & $>125$ & 1.28 & 0.02 & 3 & $9 \mathrm{H}-4,120-125$ & 80.70 & M. aragonensis & $300-355$ \\
\hline $12 \mathrm{H}-1,120-125$ & 104.70 & Cibicidoides sp. & $>125$ & 1.47 & 0.08 & 1 & $9 \mathrm{H}-5,18-19$ & 81.18 & M. aragonensis & $300-355$ \\
\hline $12 \mathrm{H}-3,120-125$ & 107.70 & Cibicidoides sp. & $>125$ & 1.69 & 0.17 & 3 & $9 \mathrm{H}-5,70-72$ & 81.70 & M. aragonensis & $300-355$ \\
\hline $3 \mathrm{H}-5,120-125$ & 25.20 & N. truempyi & & & & & $9 \mathrm{H}-5,120-125$ & 82.20 & M. aragonensis & $300-355$ \\
\hline $3 \mathrm{H}-6,120-125$ & & & $>125$ & 0.35 & 0.92 & 3 & $9 \mathrm{H}-6,6-11$ & 82.56 & M. aragonensis & $300-355$ \\
\hline $\begin{array}{l}3 \mathrm{H}-6,120-125 \\
4 \mathrm{H}-2\end{array}$ & 26.70 & $\begin{array}{l}\text { N. truempyi } \\
N \text {. truempyi }\end{array}$ & $>125$ & -0.08 & 0.98 & 3 & $9 \mathrm{H}-6,20-22$ & 82.70 & M. aragonensis & $300-355$ \\
\hline & 30.20 & & $>125$ & 0.13 & 0.84 & 3 & $9 \mathrm{H}-6,70-72$ & 83.20 & M. aragonensis & $300-355$ \\
\hline $4 \mathrm{H}-6,20-25$ & 35.20 & N. truempyi & $>125$ & 0.07 & 0.58 & 3 & $10 \mathrm{H}-1,4-6$ & 84.54 & M. aragonensis & $300-355$ \\
\hline $5 \mathrm{H}-2,120-125$ & 39.70 & N. truempyi & $>125$ & 0.13 & 0.63 & 3 & $10 \mathrm{H}-1,83-85$ & 85.33 & M. aragonensis & $300-355$ \\
\hline $5 \mathrm{H}-4,120-125$ & 42.70 & N. truempyi & $>125$ & 0.27 & 0.60 & 3 & $10 \mathrm{H}-2,4-6$ & 86.04 & M. aragonensis & $300-355$ \\
\hline $5 \mathrm{H}-6,44-49$ & 44.44 & N. truempyi & $>125$ & 0.14 & 0.68 & 3 & $10 \mathrm{H}-2,60-62$ & 86.60 & M. aragonensis & $>355$ \\
\hline $6 \mathrm{H}-2,120-125$ & 49.20 & N. truempyi & $>125$ & 0.13 & 0.41 & 3 & $10 \mathrm{H}-2,120-125$ & 87.20 & M. aragonensis & $300-355$ \\
\hline $6 \mathrm{H}-4,120-125$ & 52.20 & N. truempyi & $>125$ & 0.34 & 0.51 & 3 & $10 \mathrm{H}-3,4-6$ & 87.54 & M. aragonensis & $>355$ \\
\hline $6 \mathrm{H}-6,111-116$ & 55.11 & N. truempyi & $>125$ & 0.20 & 0.29 & 3 & $10 \mathrm{H}-2,60-62$ & 86.60 & M. subbotinae & $300-355$ \\
\hline $7 \mathrm{H}-2,120-125$ & 58.70 & N. truempyi & $>125$ & 0.00 & 0.45 & 3 & $10 \mathrm{H}-2,120-125$ & 87.20 & M. subbotinae & $300-355$ \\
\hline $7 \mathrm{H}-4,120-125$ & $\begin{array}{r}61.70 \\
63.70\end{array}$ & N. truempyi & $>125$ & 0.13 & 0.47 & 3 & $10 \mathrm{H}-3,4-6$ & 87.54 & M. subbotinae & $300-355$ \\
\hline $\begin{array}{l}7 \mathrm{H}-6,79-84 \\
8 \mathrm{H}-2,120-125\end{array}$ & $\begin{array}{l}63.79 \\
68.20\end{array}$ & $\begin{array}{l}\text { N. truempyi } \\
\text { N. truempyi }\end{array}$ & $\begin{array}{l}>125 \\
>125\end{array}$ & $\begin{array}{r}-0.01 \\
0.04\end{array}$ & $\begin{array}{l}0.43 \\
0.26\end{array}$ & $\begin{array}{l}3 \\
3\end{array}$ & $10 \mathrm{H}-3,60-62$ & 88.10 & M. subbotinae & $300-355$ \\
\hline $8 \mathrm{H}-4,120-125$ & 71.20 & N. truempyi & $>125$ & 0.67 & $\begin{array}{r}0.26 \\
-0.21\end{array}$ & $\begin{array}{l}3 \\
1\end{array}$ & $10 \mathrm{H}-3,120-125$ & 88.70 & M. subbotinae & $300-355$ \\
\hline $8 \mathrm{H}-4,120-125 \mathrm{D}$ & 71.20 & N. truempyi & $>125$ & 0.08 & 0.22 & 1 & $10 \mathrm{H}-4,4-6$ & 89.04 & M. subbotinae & $300-355$ \\
\hline $8 \mathrm{H}-6,106-111$ & 74,06 & $N$.truempyi & $>125$ & 0.03 & -0.44 & 3 & $10 \mathrm{H}-4,60-62$ & 89.60 & M. subbotinae & $300-355$ \\
\hline $9 \mathrm{H}-2,120-125$ & 77.70 & N. truempyi & $>125$ & 0.59 & -0.55 & 3 & $10 \mathrm{H}-4,120-125$ & 90.20 & M. subbotinae & $300-355$ \\
\hline $9 \mathrm{H}-4,120-125$ & 80.70 & N. truempyi & $>125$ & 0.62 & -0.56 & 3 & $10 \mathrm{H}-5,4-6$ & 90.54 & M. subbotinae & $300-355$ \\
\hline $9 \mathrm{H}-6,6-11$ & 82.56 & N.truempyi & $>125$ & -0.02 & -0.56 & 3 & $10 \mathrm{H}-5,60-62$ & 91.10 & M. subbotinae & $300-355$ \\
\hline $10 \mathrm{H}-2,120-125$ & 87.70 & N. truempyi & $>125$ & -0.17 & -0.45 & 3 & $11 \mathrm{H}-1,20-22$ & 94.20 & M. subbotinae & $300-355$ \\
\hline $10 \mathrm{H}-5,111-116$ & 91.61 & N. truempyi & $>125$ & -0.15 & -0.45 & $\overrightarrow{1}$ & $11 \mathrm{H}-1,85-87$ & 94.85 & M. subbotinae & $300-355$ \\
\hline $11 \mathrm{H}-2,120-125$ & 96.70 & N. truempyi & $>125$ & 0.22 & -0.16 & 3 & $11 \mathrm{H}-1,120-125$ & 95.20 & M. subbotinae & $300-355$ \\
\hline $11 \mathrm{H}-6,47-49$ & 101.97 & N. truempyi & $>125$ & 1.09 & -0.18 & 1 & $11 \mathrm{H}-2,20-22$ & 95.70 & M. subbotinae & $300-355$ \\
\hline $11 \mathrm{H}-\mathrm{CC}, 9-12$ & 102.53 & N. truempyi & $>125$ & 0.39 & -0.13 & 3 & $11 \mathrm{H}-2,85-87$ & 96.35 & M. subbotinae & $300-355$ \\
\hline $12 \mathrm{H}-1,120-125$ & 104.70 & N. truempyi & $>125$ & 0.93 & -0.36 & 1 & $11 \mathrm{H}-2,120-125$ & 96.70 & M. subbotinae & $300-355$ \\
\hline $12 \mathrm{H}-3,120-125$ & 107.70 & N. truempyi & $>125$ & 1.34 & 0.26 & 3 & $11 \mathrm{H}-3,20-22$ & 97.20 & M. subbotinae & $300-355$ \\
\hline $13 \mathrm{H}-, 117-121$ & 114.17 & N. truempyi & $>125$ & 1.77 & 0.13 & 1 & $11 \mathrm{H}-3,85-87$ & 97.85 & M. subbotinae & $300-355$ \\
\hline $15 X-1,120-125$ & 133.20 & N. truempyi & $>125$ & 0.27 & -0.64 & 1 & $11 \mathrm{H}-3,120-125$ & 98.20 & M. subbotinae & $300-355$ \\
\hline & & & & & & & $11 \mathrm{H}-4,20-22$ & 98.70 & M. subbotinae & $300-355$ \\
\hline $\begin{array}{l}12 \mathrm{H}-3,120-125 \\
13 \mathrm{H}-1,117-121\end{array}$ & 107.70 & Gavelinella bec. & $>125$ & 1.71 & 0.13 & 3 & $11 \mathrm{H}-4,85-87$ & 99.35 & M. subbotinae & $300-355$ \\
\hline $14 \mathrm{H}-1,120-125$ & 114.17 & Gavelinella bec. & $>125$ & 1.75 & -0.02 & 3 & $11 \mathrm{H}-4,120-125$ & 99.70 & M. subbotinae & $300-355$ \\
\hline $14 \mathrm{H}-1,120-125$ & 123.70 & Gavelinella bec. & $>125$ & 1.87 & 0.25 & 3 & $11 \mathrm{H}-5,20-22$ & 100.20 & M. subbotinae & $300-355$ \\
\hline $15 X-1,120-125$ & 133.20 & Gavelinella bec. & $>125$ & 0.68 & 0.33 & 3 & $11 \mathrm{H}-5,85-87$ & 100.85 & M. subbotinae & $300-355$ \\
\hline $15 X-3,52-57$ & 135.52 & Gavelinella bec. & $>125$ & 0.50 & -0.13 & 3 & $11 \mathrm{H}-5,111-116$ & 101.11 & M. subbotinae & $300-355$ \\
\hline Planktonic foraminif & fers & & & & & & $11 \mathrm{H}-5,120-125$ & 101.20 & M. subbotinae & $300-355$ \\
\hline $3 \mathrm{H}-1,70-72$ & 18.70 & & & & & & $11 \mathrm{H}-6,20-22$ & 101.70 & M. subbotinae & $300-355$ \\
\hline $3 \mathrm{H}-2,84-86$ & 20.34 & M. lehneri & $\begin{array}{l}300-355 \\
300-355\end{array}$ & $\begin{array}{l}2.60 \\
2.61\end{array}$ & $\begin{array}{l}-0.30 \\
-0.23\end{array}$ & $\begin{array}{l}3 \\
3\end{array}$ & $11 \mathrm{H}-6,43-47$ & 101.93 & M. subbotinae & $300-355$ \\
\hline $3 \mathrm{H}-3,78-80$ & 21.78 & M. lehneri & $300-355$ & 2.69 & -0.43 & 3 & $11 \mathrm{H}-6,85-87$ & 102.35 & M. subbotinae & $300-355$ \\
\hline $3 \mathrm{H}-4,80-82$ & 23.30 & M. lehneri & $300-355$ & 2.84 & -0.46 & 3 & $11 \mathrm{H}-\mathrm{CC}, 9-12$ & 102.53 & M. subbotinae & $300-355$ \\
\hline $3 \mathrm{H}-5,69-71$ & 24.69 & M. lehneri & $300-355$ & 2.83 & $\begin{array}{l}-0.46 \\
-0.47\end{array}$ & $\begin{array}{l}3 \\
3\end{array}$ & $12 \mathrm{H}-1,70-72$ & 104.20 & M. subbotinae & $300-355$ \\
\hline $3 \mathrm{H}-6,69-71$ & 26.19 & M. lehneri & $300-355$ & 2.72 & -0.50 & 3 & $11 \mathrm{H}-5,85-87$ & 100.85 & M. velascoensis & $300-355$ \\
\hline $3 \mathrm{H}-7,12-14$ & 27.12 & M. lehneri & $300-355$ & 2.69 & -0.40 & 3 & $11 \mathrm{H}-6,20-22 \mathrm{D}$ & 101.70 & M. velascoensis & $300-355$ \\
\hline $4 \mathrm{H}-1,70-72$ & 28.20 & M. lehneri & $300-355$ & 2.71 & -0.59 & 3 & $11 \mathrm{H}-6,20-22$ & 101.70 & M. velascoensis & $300-355$ \\
\hline $4 \mathrm{H}-2,84-86$ & 29.84 & M. lehneri & $300-355$ & 3.01 & -0.63 & 3 & $11 \mathrm{H}-6,85-87$ & 102.35 & M. velascoensis & $300-355$ \\
\hline $4 \mathrm{H}-3,84-86$ & 31.34 & M. lehneri & $300-355$ & 3.04 & -0.70 & 3 & $11 \mathrm{H}-\mathrm{CC}, 9-12$ & 102.53 & M. velascoensis & $300-355$ \\
\hline $4 \mathrm{H}-4,70-72$ & 32.70 & M. lehneri & $300-355$ & 2.74 & -0.65 & 3 & $12 \mathrm{H}-1,0-2$ & 103.50 & M. velascoensis & $300-355$ \\
\hline $4 \mathrm{H}-5,70-72$ & 34.20 & M. lehneri & $300-355$ & 2.62 & -0.54 & 3 & $12 \mathrm{H}-1,2-4$ & 103.52 & M. velascoensis & $300-355$ \\
\hline $4 \mathrm{H}-6,20-22$ & 35.20 & M. lehneri & $300-355$ & 2.95 & -0.62 & 3 & $12 \mathrm{H}-1,10-12$ & 103.60 & M. velascoensis & $300-355$ \\
\hline $5 \mathrm{H}-1,70-72$ & 37.70 & M. lehneri & $300-355$ & 2.99 & -0.49 & 3 & $12 \mathrm{H}-1,12-14$ & 103.62 & M. velascoensis & $300-355$ \\
\hline $5 \mathrm{H}-2,70-72$ & 39.20 & M. lehneri & $300-355$ & 2.63 & -0.47 & 3 & $12 \mathrm{H}-1,20-22 \mathrm{D}$ & 103.70 & M. velascoensis & $300-355$ \\
\hline $5 \mathrm{H}-3,70-72$ & 40.70 & M. lehneri & $300-355$ & 2.70 & -0.50 & 3 & $12 \mathrm{H}-1,20-22 \mathrm{D}$ & 103.70 & M. velascoensis & $300-355$ \\
\hline $5 \mathrm{H}-4,70-72$ & 42.20 & M. lehneri & $300-355$ & 2.89 & -0.64 & 3 & $12 \mathrm{H}-1,20-22$ & 103.70 & M. velascoensis & $300-355$ \\
\hline $5 \mathrm{H}-5,70-72$ & 43.70 & M. lehneri & $300-355$ & 2.85 & -0.71 & 3 & $12 \mathrm{H}-1,30-32$ & 103.80 & M. velascoensis & $300-355$ \\
\hline $5 \mathrm{H}-6,50-52$ & 44.50 & M. lehneri & $300-355$ & 2.78 & -0.68 & 3 & $12 \mathrm{H}-1,30-32 \mathrm{D}$ & 103.80 & M. velascoensis & $300-355$ \\
\hline $5 \mathrm{H}-6,50-52 \mathrm{D}$ & 44.50 & M. lehneri & $300-355$ & 2.80 & -0.69 & 3 & $12 \mathrm{H}-1,40-42$ & 103.90 & M. velascoensis & $300-355$ \\
\hline $6 \mathrm{H}-1,81-83$ & 47.31 & M. lehneri & $300-355$ & 2.94 & -0.71 & 3 & $12 \mathrm{H}-1,40-42 \mathrm{D}$ & 103.90 & M. velascoensis & $300-355$ \\
\hline $6 \mathrm{H}-2,73-75$ & 48.73 & M. lehneri & $300-355$ & 2.78 & -0.46 & 3 & $12 \mathrm{H}-1,70-72 \mathrm{D}$ & 104.20 & M. velascoensis & $300-355$ \\
\hline $6 \mathrm{H}-3,70-72$ & 50.20 & M. lehneri & $300-355$ & 2.89 & -0.76 & 3 & $12 \mathrm{H}-1,70-72 \mathrm{D}$ & 104.20 & M. velascoensis & $300-355$ \\
\hline $6 \mathrm{H}-4,70-72$ & 51.70 & M. lehneri & $300-355$ & 2.97 & -0.76 & 3 & $12 \mathrm{H}-1,70-72$ & 104.20 & M. velascoensis & $300-355$ \\
\hline $6 \mathrm{H}-5,70-72$ & 53.20 & M. lehneri & $300-355$ & 3.05 & -0.73 & 3 & $12 \mathrm{H}-1,120-125 \mathrm{D}$ & 104.70 & M. velascoensis & $300-355$ \\
\hline $6 \mathrm{H}-6,70-72$ & 54.70 & M. lehneri & $300-355$ & 3.20 & -1.04 & 3 & $12 \mathrm{H}-1,120-125$ & 104.70 & M. velascoensis & $300-355$ \\
\hline
\end{tabular}


Table 1 (continued).

\begin{tabular}{|c|c|c|c|c|c|c|c|c|c|}
\hline $\begin{array}{l}\text { Core, section, } \\
\text { interval }(\mathrm{cm})\end{array}$ & $\begin{array}{l}\text { Depth } \\
\text { (mbsf) }\end{array}$ & Taxon & $\begin{array}{l}\text { Size } \\
(\mu \mathrm{m})\end{array}$ & $\begin{array}{l}\delta^{13} \mathrm{C} \\
(\% \infty)\end{array}$ & $\begin{array}{l}\delta^{18} \mathrm{O} \\
(\% 00)\end{array}$ & Run & $\begin{array}{l}\text { Core, section, } \\
\text { interval }(\mathrm{cm})\end{array}$ & $\begin{array}{l}\text { Depth } \\
\text { (mbsf) }\end{array}$ & Taxon \\
\hline $12 \mathrm{H}-2,20-22$ & 105.20 & M. velascoensis & $300-355$ & 3.91 & -1.75 & 3 & $8 \mathrm{H}-4,70-72$ & 70.70 & Subbotina spp. \\
\hline $12 \mathrm{H}-2,70-72 \mathrm{D}$ & 105.70 & M. velascoensis & $300-355$ & 4.55 & -1.81 & 3 & $8 \mathrm{H}-5,70-72$ & 72.20 & Subbotina spp. \\
\hline $12 \mathrm{H}-2,70-72$ & 105.70 & M. velascoensis & $300-355$ & 4.20 & -1.80 & 1 & $8 \mathrm{H}-6,70-72$ & 73.70 & Subbotina spp. \\
\hline $12 \mathrm{H}-2,120-125$ & 106.20 & M. velascoensis & $300-355$ & 4.38 & -1.96 & $i$ & $9 \mathrm{H}-1,13-15$ & 75.13 & Subbotina spp. \\
\hline $12 \mathrm{H}-3,20-22$ & 106.70 & M. velascoensis & $300-355$ & 4.39 & -1.75 & 3 & $9 \mathrm{H}-1,70-72$ & 75.70 & Subbotina spp. \\
\hline $12 \mathrm{H}-3,70-72$ & 107.20 & M. velascoensis & $300-355$ & 4.36 & -1.73 & 1 & $9 \mathrm{H}-1,120-125$ & 76.20 & Subbotina spp. \\
\hline $12 \mathrm{H}-3,120-125$ & 107.70 & M. velascoensis & $300-355$ & 4.78 & -1.81 & $i$ & $9 \mathrm{H}-2,20-22$ & 76.70 & Subbotina spp. \\
\hline $12 \mathrm{H}-4,70-72$ & 108.70 & M. velascoensis & $300-355$ & 4.50 & -1.82 & 1 & $9 \mathrm{H}-2,70-72$ & 77.20 & Subbotina spp. \\
\hline $12 \mathrm{H}-4,120-125$ & 109.20 & M. velascoensis & $300-355$ & 4.54 & -1.91 & $i$ & $9 \mathrm{H}-2,120-125$ & 77.70 & Subbotina spp. \\
\hline $12 \mathrm{H}-5,20-22$ & 109.70 & M. velascoensis & $300-355$ & 4.52 & -1.76 & 3 & $9 \mathrm{H}-3,20-22$ & 78.20 & Subbotina spp. \\
\hline $12 \mathrm{H}-5,70-72$ & 110.20 & M. velascoensis & $300-355$ & 4.45 & -2.31 & 1 & $9 \mathrm{H}-3,70-72$ & 78.70 & Subbotina spp. \\
\hline $12 \mathrm{H}-5,120-125$ & 110.70 & M. velascoensis & $300-355$ & 4.78 & -1.81 & 1 & $9 \mathrm{H}-3,120-125$ & 79.20 & Subbotina spp. \\
\hline $12 \mathrm{H}-6,20-22$ & 111.20 & M. velascoensis & $300-355$ & 4.77 & -1.87 & i & $9 \mathrm{H}-4,10-12$ & 79.60 & Subbotina spp. \\
\hline $12 \mathrm{H}-6,70-72$ & 111.70 & M. velascoensis & $300-355$ & 5.01 & -1.75 & $i$ & $9 \mathrm{H}-4,70-72$ & 80.20 & Subbotina spp. \\
\hline $12 \mathrm{H}-6,102-104$ & 112.02 & M. velascoensis & $300-355$ & 4.75 & -1.70 & $i$ & $9 \mathrm{H}-4,120-125$ & 80.70 & Subbotina spp. \\
\hline $13 \mathrm{H}-1,21-23 \mathrm{D}$ & 113.21 & M. velascoensis & $300-355$ & 4.58 & -1.87 & 3 & $9 \mathrm{H}-5,18-19$ & 81.18 & Subbotina spp. \\
\hline $13 \mathrm{H}-1,21-23 \mathrm{D}$ & 113.21 & M. velascoensis & $300-355$ & 4.59 & -1.82 & 1 & $9 \mathrm{H}-5,70-72$ & 81.70 & Subbotina spp. \\
\hline $13 \mathrm{H}-1,21-23$ & 113.21 & M. velascoensis & $300-355$ & 4.63 & -1.76 & 1 & $9 \mathrm{H}-5,70-72 \mathrm{D}$ & 81.70 & Subbotina spp. \\
\hline $13 \mathrm{H}-1,70-72$ & 113.70 & M. velascoensis & $300-355$ & 4.50 & -1.80 & 1 & $9 \mathrm{H}-5,120-125$ & 82.20 & Subbotina spp. \\
\hline $13 \mathrm{H}-1,117-121$ & 114.17 & M. velascoensis & $300-355$ & 4.86 & -1.71 & 3 & $9 \mathrm{H}-6,20-22$ & 82.70 & Subbotina spp. \\
\hline $13 \mathrm{H}-2,17-19 \mathrm{D}$ & 114.67 & M. velascoensis & $300-355$ & 4.79 & -1.66 & 3 & $9 \mathrm{H}-6,70-72$ & 83.20 & Subbotina spp. \\
\hline $13 \mathrm{H}-2,17-19$ & 114.67 & $M$. velascoensis & $300-355$ & 4.88 & -1.57 & 1 & $10 \mathrm{H}-1,4-6$ & 84.54 & Subbotina spp. \\
\hline $13 \mathrm{H}-2,70-72$ & 115.20 & M. velascoensis & $300-355$ & 4.64 & -1.59 & i & $10 \mathrm{H}-1,83-85$ & 85.33 & Subbotina spp. \\
\hline $13 \mathrm{H}-2,123-128$ & 115.73 & M. velascoensis & $300-355$ & 4.55 & -1.73 & 3 & $10 \mathrm{H}-1,120-125$ & 85.70 & Subbotina spp. \\
\hline $13 \mathrm{H}-3,21-23$ & 116.21 & $M$. velascoensis & $300-355$ & 4.52 & -1.92 & 1 & $10 \mathrm{H}-2,4-6$ & 86.04 & Subbotina spp. \\
\hline $13 \mathrm{H}-3,70-72$ & 116.70 & $M$. velascoensis & $300-355$ & 4.86 & -1.98 & 1 & $10 \mathrm{H}-2,60-62$ & 86.60 & Subbotina spp. \\
\hline $13 \mathrm{H}-3,121-126$ & 117.21 & M. velascoensis & $300-355$ & 4.93 & -1.79 & 3 & $10 \mathrm{H}-2,120-125$ & 87.20 & Subbotina spp. \\
\hline $13 \mathrm{H}-4,18-20$ & 117.68 & $M$. velascoensis & $300-355$ & 5.12 & -1.74 & 1 & $10 \mathrm{H}-3,4-6$ & 87.54 & Subbotina spp. \\
\hline $13 \mathrm{H}-4,70-72$ & 118.20 & M. velascoensis & $300-355$ & 4.80 & -1.67 & $i$ & $10 \mathrm{H}-3,60-62$ & 88.10 & Subbotina spp. \\
\hline $13 \mathrm{H}-4,123-128$ & 118.73 & M. velascoensis & $300-355$ & 4.71 & -1.68 & 3 & $10 \mathrm{H}-3,120-125$ & 88.70 & Subbotina spp. \\
\hline $13 \mathrm{H}-5,20-22$ & 119.20 & M. velascoensis & $300-355$ & 4.87 & -1.63 & 1 & $10 \mathrm{H}-4,4-6$ & 89.04 & Subbotina spp. \\
\hline $13 \mathrm{H}-5,70-72$ & 119.70 & $M$. velascoensis & $300-355$ & 4.74 & -1.60 & 1 & $10 \mathrm{H}-4,60-62$ & 89.60 & Subbotina spp. \\
\hline $13 \mathrm{H}-5,123-128$ & 120.23 & M. velascoensis & $300-355$ & 4.95 & -1.86 & 3 & $10 \mathrm{H}-4,120-125$ & 90.20 & Subbotina spp. \\
\hline $13 \mathrm{H}-6,70-72$ & 121.20 & M. velascoensis & $300-355$ & 4.42 & -1.64 & 1 & $10 \mathrm{H}-5,4-6$ & 90.54 & Subbotina spp. \\
\hline $13 \mathrm{H}-6,89-93$ & 121.39 & M. velascoensis & $300-355$ & 4.27 & -1.47 & 3 & $10 \mathrm{H}-5,60-62$ & 91.10 & Subbotina spp. \\
\hline $14 \mathrm{H}-1,20-22$ & 122.70 & $M$. velascoensis & $300-355$ & 4.48 & -1.58 & 1 & $10 \mathrm{H}-5,111-116$ & 91.61 & Subbotina spp. \\
\hline $14 \mathrm{H}-1,70-72$ & 123.20 & M. velascoensis & $300-355$ & 4.42 & -1.63 & i & $11 \mathrm{H}-1,20-22$ & 94.20 & Subbotina spp. \\
\hline $14 \mathrm{H}-1,130-132$ & 123.80 & $M$. velascoensis & $300-355$ & 4.33 & -1.29 & $i$ & $11 \mathrm{H}-1,85-87$ & 94.85 & Subbotina spp. \\
\hline $14 \mathrm{H}-1,144-146$ & 123.94 & M. velascoensis & $300-355$ & 4.24 & -1.65 & 3 & $11 \mathrm{H}-2,20-22$ & 95.70 & Subbotina spp. \\
\hline $14 \mathrm{H}-2,20-22$ & 124.20 & M. velascoensis & $300-355$ & 4.30 & -1.44 & 1 & $11 \mathrm{H}-2,85-87$ & 96.35 & Subbotina spp. \\
\hline $14 \mathrm{H}-2,66-68$ & 124.66 & $M$. velascoensis & $300-355$ & 4.52 & -1.45 & $i$ & $11 \mathrm{H}-3,20-22$ & 97.20 & Subbotina spp. \\
\hline $14 \mathrm{H}-3,25-27$ & 125.75 & $M$. velascoensis & $300-355$ & 4.29 & -1.41 & $i$ & $11 \mathrm{H}-3,85-87$ & 97.85 & Subbotina spp. \\
\hline $14 \mathrm{H}-3,76-78$ & 126.26 & M. velascoensis & $300-355$ & 4.28 & -1.56 & 1 & $11 \mathrm{H}-4,20-22$ & 98.70 & Subbotina spp. \\
\hline $14 \mathrm{H}-3,138-140$ & 126.88 & M. velascoensis & $300-355$ & 4.03 & -1.09 & 3 & $11 \mathrm{H}-4,85-87$ & 99.35 & Subbotina spp. \\
\hline $14 \mathrm{H}-4,23-25$ & 127.23 & $M$. velascoensis & $300-355$ & 4.30 & -1.32 & 1 & $11 \mathrm{H}-5,20-22$ & 100.20 & Subbotina spp. \\
\hline $14 \mathrm{H}-4,65-67$ & 127.65 & M. velascoensis & $300-355$ & 4.30 & -1.39 & 1 & $11 \mathrm{H}-5,85-87$ & 100.85 & Subbotina spp. \\
\hline $14 \mathrm{H}-4,65-67 \mathrm{D}$ & 127.65 & $M$. velascoensis & $300-355$ & 4.19 & -1.27 & $i$ & $11 \mathrm{H}-6,20-22$ & 101.70 & Subbotina spp. \\
\hline $14 \mathrm{H}-5,22-24$ & 128.72 & M. velascoensis & $300-355$ & 3.93 & -1.50 & 1 & $11 \mathrm{H}-\mathrm{CC}, 9-12$ & 102.53 & Subbotina spp. \\
\hline $14 \mathrm{H}-5,66-68$ & 129.16 & M. velascoensis & $300-355$ & 3.83 & -1.39 & 1 & $12 \mathrm{H}-1,00-02$ & 103.50 & Subbotina spp. \\
\hline $14 \mathrm{H}-5,136-138$ & 129.86 & $M$. velascoensis & $300-355$ & 3.77 & -1.42 & 3 & $12 \mathrm{H}-1,10-12$ & 103.60 & Subbotina spp. \\
\hline & & & & & & & $12 \mathrm{H}-1,20-22$ & 103.70 & Subbotina spp. \\
\hline $3 \mathrm{H}-1,70-72$ & 18.70 & Subbotina spp. & $300-355$ & 1.32 & 0.82 & 3 & $12 \mathrm{H}-1,30-32$ & 103.80 & Subbotina spp. \\
\hline $3 \mathrm{H}-2,84-86$ & 20.34 & Subbotina spp. & $300-355$ & 1.30 & 0.73 & 3 & $12 \mathrm{H}-1,40-42$ & 103.90 & Subbotina spp. \\
\hline $3 \mathrm{H}-3,78-80$ & 21.78 & Subbotina spp. & $300-355$ & 1.42 & 0.50 & 3 & $12 \mathrm{H}-1,70-72$ & 104.20 & Subbotina spp. \\
\hline $3 \mathrm{H}-4,80-82$ & 23.30 & Subbotina spp. & $300-355$ & 1.56 & 0.84 & 3 & $12 \mathrm{H}-1,120-125$ & 104.70 & Subbotina spp. \\
\hline $3 \mathrm{H}-5,69-71$ & 24.69 & Subbotina spp. & $300-355$ & 1.49 & 1.21 & 3 & $12 \mathrm{H}-2,20-22$ & 105.20 & Subbotina spp. \\
\hline $3 \mathrm{H}-6,69-71$ & 26.19 & Subbotina spp. & $300-355$ & 1.34 & 0.70 & 3 & $12 \mathrm{H}-2,70-72$ & 105.70 & Subbotina spp. \\
\hline $3 \mathrm{H}-7,12-14$ & 27.12 & Subbotina spp. & $300-355$ & 1.28 & 0.84 & 3 & $12 \mathrm{H}-2,120-125$ & 106.20 & Subbotina spp. \\
\hline $4 \mathrm{H}-1,70-72$ & 28.20 & Subbotina spp. & $300-355$ & 1.26 & 0.59 & 3 & $12 \mathrm{H}-3,20-22$ & 106.70 & Subbotina spp. \\
\hline $4 \mathrm{H}-1,70-72 \mathrm{D}$ & 28.20 & Subbotina spp. & $300-355$ & 1.22 & 0.72 & 3 & $12 \mathrm{H}-3,70-72$ & 107.20 & Subbotina spp. \\
\hline $4 \mathrm{H}-2,84-86$ & 29.84 & Subbotina spp. & $300-355$ & 1.34 & 0.46 & 3 & $12 \mathrm{H}-3,120-125$ & 107.70 & Subbotina spp. \\
\hline $4 \mathrm{H}-3,84-86$ & 31.34 & Subbotina spp. & $300-355$ & 1.38 & 0.58 & 3 & $12 \mathrm{H}-4,70-72$ & 108.70 & Subbotina spp. \\
\hline $4 \mathrm{H}-4,70-72$ & 32.70 & Subbotina spp. & $300-355$ & 1.62 & 0.26 & 3 & $12 \mathrm{H}-4,120-125$ & 109.20 & Subbotina spp. \\
\hline $4 \mathrm{H}-5,70-72$ & 34.20 & Subbotina spp. & $300-355$ & 1.51 & 0.19 & 3 & $12 \mathrm{H}-5,20-22$ & 109.70 & Subbotina spp. \\
\hline $4 \mathrm{H}-6,20-22$ & 35.20 & Subbotina spp. & $300-355$ & 1.63 & -0.08 & 3 & $12 \mathrm{H}-5,70-72$ & 110.20 & Subbotina spp. \\
\hline $5 \mathrm{H}-1,70-72$ & 37.70 & Subbotina spp. & $300-355$ & 1.66 & -0.02 & 3 & $12 \mathrm{H}-5,120-125$ & 110.70 & Subbotina spp. \\
\hline $5 \mathrm{H}-2,70-72$ & 39.20 & Subbotina spp. & $255-300$ & 1.66 & -0.06 & 3 & $12 \mathrm{H}-6,20-22$ & 111.20 & Subbotina spp. \\
\hline $5 \mathrm{H}-3,70-72$ & 40.70 & Subbotina spp. & $300-355$ & 1.56 & -0.14 & 3 & $12 \mathrm{H}-6,70-72$ & 111.70 & Subbotina spp. \\
\hline $5 \mathrm{H}-4,70-72$ & 42.20 & Subbotina spp. & $300-355$ & 1.63 & 0.12 & 3 & $12 \mathrm{H}-6,102-104$ & 112.02 & Subbotina spp. \\
\hline $5 \mathrm{H}-5,70-72$ & 43.70 & Subbotina spp. & $300-355$ & 1.68 & -0.02 & 3 & $13 \mathrm{H}-1,21-23$ & 113.21 & Subbotina spp. \\
\hline $5 \mathrm{H}-6,50-52$ & 44.50 & Subbotina spp. & $300-355$ & 1.62 & -0.09 & 3 & $13 \mathrm{H}-1,70-72$ & 113.70 & Subbotina spp. \\
\hline $6 \mathrm{H}-1,81-83$ & 47,31 & Subbotina spp. & $300-355$ & 1.53 & -0.11 & 3 & $13 \mathrm{H}-1,117-121$ & 114.17 & Subbotina spp. \\
\hline $6 \mathrm{H}-2,73-75$ & 48.73 & Subbotina spp. & $300-355$ & 1.56 & -0.01 & 3 & $13 \mathrm{H}-2,17-19$ & 114.67 & Subbotina spp. \\
\hline $6 \mathrm{H}-3,70-72$ & 50.20 & Subbotina spp. & $300-355$ & 1.84 & 0.00 & 3 & $13 \mathrm{H}-2,70-72$ & 115.20 & Subbotina spp. \\
\hline $6 \mathrm{H}-4,70-72$ & 51.70 & Subbotina spp. & $300-355$ & 1.81 & -0.13 & 3 & $13 \mathrm{H}-2,123-128$ & 115.73 & Subbotina spp. \\
\hline $6 \mathrm{H}-5,70-72$ & 53.20 & Subbotina spp. & $300-355$ & 1.76 & 0.05 & 3 & $13 \mathrm{H}-3,21-23$ & 116.21 & Subbotina spp. \\
\hline $6 \mathrm{H}-6,70-72$ & 54.70 & Subbotina spp. & $300-355$ & 1.58 & -0.01 & 3 & $13 \mathrm{H}-3,70-72$ & 116.70 & Subbotina spp. \\
\hline $7 \mathrm{H}-1,118-120$ & 57.18 & Subbotina spp. & $300-355$ & 1.61 & 0.04 & 3 & $13 \mathrm{H}-3,121-126$ & 117.21 & Subbotina spp. \\
\hline $7 \mathrm{H}-2,78-80$ & 58.28 & Subbotina spp. & $300-355$ & 1.71 & 0.15 & 3 & $13 \mathrm{H}-4,18-20$ & 117.68 & Subbotina spp. \\
\hline $7 \mathrm{H}-2,78-80 \mathrm{D}$ & 58.28 & Subbotina spp. & $300-355$ & 1.51 & 0.30 & 3 & $13 \mathrm{H}-4,70-72$ & 118.20 & Subbotina spp. \\
\hline $7 \mathrm{H}-3,69-71$ & 59.69 & Subbotina spp. & $300-355$ & 1.51 & 0.09 & 3 & $13 \mathrm{H}-4,123-128$ & 118.73 & Subbotina spp. \\
\hline $7 \mathrm{H}-4,68-70$ & 61.18 & Subbotina spp. & $300-355$ & 1.62 & 0.03 & 3 & $13 \mathrm{H}-5,20-22$ & 119.20 & Subbotina spp. \\
\hline $7 \mathrm{H}-5,70-72$ & 62.70 & Subbotina spp. & $300-355$ & 2.11 & -0.53 & 3 & $13 \mathrm{H}-5,70-72$ & 119.70 & Subbotina spp. \\
\hline $7 \mathrm{H}-6,76-78$ & 63.76 & Subbotina spp. & $300-355$ & 2.12 & -0.54 & 3 & $13 \mathrm{H}-5,123-128$ & 120.23 & Subbotina spp. \\
\hline $8 \mathrm{H}-1,89-91$ & 66.39 & Subbotina spp. & $300-355$ & 1.92 & -0.32 & 3 & $13 \mathrm{H}-6,20-22$ & 120.70 & Subbotina spp. \\
\hline $8 \mathrm{H}-2,70-72$ & 67.70 & Subbotina spp. & $300-355$ & 1.55 & -0.27 & 3 & $13 \mathrm{H}-6,89-93$ & 121.39 & Subbotina spp. \\
\hline $8 \mathrm{H}-3,70-72$ & 69.20 & Subbotina spp. & $300-355$ & 1.44 & 0.07 & 3 & $14 \mathrm{H}-1,130-132$ & 123.80 & Subbotina spp. \\
\hline $8 \mathrm{H}-4,67-69$ & 70.67 & Subbotina spp. & $300-355$ & 1.27 & 0.08 & 3 & $14 \mathrm{H}-1,144-146$ & 123.94 & Subbotina spp. \\
\hline
\end{tabular}


Table 1 (continued).

\begin{tabular}{|c|c|c|c|c|c|c|c|c|c|c|c|c|c|}
\hline $\begin{array}{l}\text { Core, section, } \\
\text { interval }(\mathrm{cm})\end{array}$ & $\begin{array}{l}\text { Depth } \\
\text { (mbsf) }\end{array}$ & Taxon & $\begin{array}{l}\text { Size } \\
(\mu \mathrm{m})\end{array}$ & $\begin{array}{l}\delta^{13} \mathrm{C} \\
(\%)\end{array}$ & $\begin{array}{l}\delta^{18} \mathrm{O} \\
(\%)\end{array}$ & Run & $\begin{array}{l}\text { Core, section, } \\
\text { interval }(\mathrm{cm})\end{array}$ & $\begin{array}{l}\text { Depth } \\
\text { (mbsf) }\end{array}$ & Taxon & $\begin{array}{l}\text { Size } \\
(\mu \mathrm{m})\end{array}$ & $\begin{array}{l}\delta^{13} \mathrm{C} \\
(\%))\end{array}$ & $\begin{array}{l}\delta^{18} \mathrm{O} \\
(\% \circ)\end{array}$ & Run \\
\hline $14 \mathrm{H}-2,66-68$ & 124.66 & Subbotina spp. & $300-355$ & 2.45 & 0.13 & 1 & $10 \mathrm{H}-1,83-85$ & 85.33 & A. soldadoensis & $300-355$ & 2.55 & -1.96 & 3 \\
\hline $14 \mathrm{H}-3,76-78$ & 126.26 & Subbotina spp. & $250-300$ & 2.29 & 0.02 & 1 & $10 \mathrm{H}-1,120-125$ & 85.70 & A. soldadoensis & $300-355$ & 2.49 & -2.02 & 3 \\
\hline $14 \mathrm{H}-3,138-140$ & 126.88 & Subbotina spp. & $300-355$ & 2.20 & 0.14 & 3 & $10 \mathrm{H}-2,4-6$ & 86.04 & A. soldadoensis & $300-355$ & 2.52 & -1.93 & 3 \\
\hline $14 \mathrm{H}-4,65-67$ & 127.65 & Subbotina spp. & $300-355$ & 2.24 & 0.12 & 1 & $10 \mathrm{H}-2,60-62$ & 86.60 & A. soldadoensis & $300-355$ & 2.69 & -2.08 & 3 \\
\hline $14 \mathrm{H}-5,66-68$ & 129.16 & Subbotina spp. & $300-355$ & 2.51 & -0.87 & 1 & $10 \mathrm{H}-2,120-125$ & 87.20 & A. soldadoensis & $300-355$ & 2.79 & -1.84 & 3 \\
\hline $14 \mathrm{H}-5,136-138$ & 129.86 & Subbotina spp. & $300-355$ & 2.16 & -0.70 & 3 & $10 \mathrm{H}-3,4-6$ & 87.54 & A. soldadoensis & $300-355$ & 2.62 & -2.03 & 3 \\
\hline $14 \mathrm{H}-6,17-19$ & 130.17 & Subbotina spp. & $250-300$ & 2.19 & -0.60 & 3 & $10 \mathrm{H}-3,4-6 \mathrm{D}$ & 87.54 & A. soldadoensis & $300-355$ & 2.26 & -2.29 & 3 \\
\hline $15 X-1,73-75$ & 132.73 & Subbotina spp. & $250-300$ & 2.01 & -0.24 & 1 & $10 \mathrm{H}-3,60-62$ & 88.10 & A. soldadoensis & $300-355$ & 2.94 & -1.82 & 2 \\
\hline \multirow[t]{2}{*}{$15 X-2,21-23$} & 133.71 & Subbotina spp. & $250-300$ & 1.81 & -0.20 & $i$ & $10 \mathrm{H}-3,120-125$ & 88.70 & A. soldadoensis & $300-355$ & 3.20 & -1.87 & 2 \\
\hline & & & & & & & $10 \mathrm{H}-4,4-6$ & 89.04 & A. soldadoensis & $300-355$ & 2.91 & -1.94 & \\
\hline $3 \mathrm{H}-1,70-72$ & 18.70 & A. bullbrooki & $300-355$ & 1.48 & 0.20 & 3 & $10 \mathrm{H}-4,60-62$ & 89.60 & A. soldadoensis & $300-355$ & 3.06 & -1.94 & 2 \\
\hline $3 \mathrm{H}-2,84-86$ & 20.34 & A. bullbrooki & $300-355$ & 1.61 & 0.25 & 3 & $10 \mathrm{H}-4,120-125$ & 90.20 & A. soldadoensis & $300-355$ & 2.96 & -1.96 & 2 \\
\hline $3 \mathrm{H}-3,78-80$ & 21.78 & A. bullbrooki & $300-355$ & 1.66 & 0.01 & 3 & $10 \mathrm{H}-5,4-6$ & 90.54 & A. soldadoensis & $300-355$ & 2.97 & -1.90 & 2 \\
\hline $3 \mathrm{H}-4,80-82$ & 23.30 & A. bullbrooki & $300-355$ & 2.08 & -0.10 & 3 & $10 \mathrm{H}-5,60-62$ & 91.10 & A. soldadoensis & $300-355$ & 2.43 & -1.87 & 3 \\
\hline $3 \mathrm{H}-5,69-71$ & 24.69 & A. bullbrooki & $300-355$ & 1.62 & 0.14 & 3 & $10 \mathrm{H}-5,111-116$ & 91.61 & A. soldadoensis & $300-355$ & 2.89 & -2.03 & 2 \\
\hline $3 \mathrm{H}-6,69-71$ & 26.19 & A. bullbrooki & $300-355$ & 2.21 & -0.27 & 3 & $11 \mathrm{H}-1,20-22$ & 94.20 & A. soldadoensis & $300-355$ & 2.98 & -2.14 & 2 \\
\hline $3 \mathrm{H}-7,12-14$ & 27.12 & A. bullbrooki & $300-355$ & 1.95 & -0.30 & 3 & $11 \mathrm{H}-1,85-87$ & 94.85 & A. soldadoensis & $300-355$ & 3.46 & -2.01 & 2 \\
\hline $4 \mathrm{H}-1,70-72$ & 28.20 & A. bullbrooki & $300-355$ & 2.85 & -0.21 & 3 & $11 \mathrm{H}-1,120-125$ & 95.20 & A. soldadoensis & $300-355$ & 3.06 & -2.06 & 3 \\
\hline $4 \mathrm{H}-2,84-86$ & 29.84 & A. bullbrooki & $300-355$ & 2.86 & -0.44 & 3 & $11 \mathrm{H}-2,20-22$ & 95.70 & A. soldadoensis & $300-355$ & 3.26 & -2.07 & 2 \\
\hline $4 \mathrm{H}-3,84-86$ & 31.34 & A. bullbrooki & $300-355$ & 3.20 & -0.59 & 3 & $11 \mathrm{H}-2,85-87$ & 96.35 & A. soldadoensis & $300-355$ & 3.39 & -2.23 & 2 \\
\hline $4 \mathrm{H}-4,70-72$ & 32.70 & A. bullbrooki & $300-355$ & 3.23 & -0.58 & 3 & $11 \mathrm{H}-2,85-87 \mathrm{D}$ & 96.35 & A. soldadoensis & $300-355$ & 3.28 & -2.15 & 2 \\
\hline $4 \mathrm{H}-5,70-72$ & 34.20 & A. bullbrooki & $300-355$ & 2.05 & -0.59 & 3 & $11 \mathrm{H}-2,120-125$ & 96.70 & A. soldadoensis & $300-355$ & 3.24 & -2.13 & 3 \\
\hline $4 \mathrm{H}-6,20-22$ & 35.20 & A. bullbrooki & $300-355$ & 1.59 & -0.48 & 3 & $11 \mathrm{H}-3,20-22$ & 97.20 & A. soldadoensis & $300-355$ & 3.68 & -2.14 & 2 \\
\hline $5 \mathrm{H}-1,70-72$ & 37.70 & A. bullbrooki & $300-355$ & 2.01 & -0.38 & 3 & $11 \mathrm{H}-3,85-87$ & 97.85 & A. soldadoensis & $300-355$ & 3.58 & -2.21 & 2 \\
\hline $5 \mathrm{H}-2,70-72$ & 39.20 & A. bullbrooki & $300-355$ & 1.59 & -0.33 & 3 & $11 \mathrm{H}-3,120-125$ & 98.20 & A. soldadoensis & $300-355$ & 3.57 & -2.26 & 3 \\
\hline $5 \mathrm{H}-3,70-72$ & 40.70 & A. bullbrooki & $300-355$ & 1.41 & -0.10 & 3 & $11 \mathrm{H}-4,20-22$ & 98.70 & A. soldadoensis & $300-355$ & 3.38 & -2.12 & 2 \\
\hline $5 \mathrm{H}-4,70-72$ & 42.20 & A. bullbrooki & $300-355$ & 2.11 & -0.30 & 3 & $11 \mathrm{H}-4,85-87$ & 99.35 & A. soldadoensis & $300-355$ & 3.75 & -2.22 & 2 \\
\hline $5 \mathrm{H}-5,70-72$ & 43.70 & A. bullbrooki & $300-355$ & 3.33 & -0.98 & 3 & $11 \mathrm{H}-4,120-125$ & 99.70 & A. soldadoensis & $300-355$ & 3.58 & -2.25 & 3 \\
\hline $5 \mathrm{H}-6,50-52$ & 44.50 & A. bullbrooki & $300-355$ & 3.08 & -0.77 & 3 & $11 \mathrm{H}-5,20-22$ & 100.20 & A. soldadoensis & $300-355$ & 3.65 & -1.80 & 2 \\
\hline $6 \mathrm{H}-1,81-83$ & 47.31 & A. bullbrooki & $300-355$ & 3.00 & -0.63 & 3 & $11 \mathrm{H}-5,85-87$ & 100.85 & A. soldadoensis & $300-355$ & 3.37 & -2.18 & 2 \\
\hline $6 \mathrm{H}-2,73-75$ & 48.73 & A. bullbrooki & $300-355$ & 1.71 & -0.26 & 3 & $11 \mathrm{H}-5,120-125$ & 101.20 & A. soldadoensis & $300-355$ & 3.23 & -2.14 & 3 \\
\hline $6 \mathrm{H}-3,70-72$ & 50.20 & A. bullbrooki & $300-355$ & 3.06 & -0.78 & 3 & $11 \mathrm{H}-6,20-22 \mathrm{D}$ & 101.70 & A. soldadoensis & $300-355$ & 3.43 & -2.16 & 3 \\
\hline $6 \mathrm{H}-4,70-72$ & 51.70 & A. bullbrooki & $300-355$ & 3.13 & -0.96 & 3 & $11 \mathrm{H}-6,20-22$ & 101.70 & A. soldadoensis & $300-355$ & 3.40 & -2.11 & 2 \\
\hline $6 \mathrm{H}-4,70-72 \mathrm{D}$ & 51.70 & A. bullbrooki & $300-355$ & 3.17 & -0.85 & 3 & $11 \mathrm{H}-6,43-47$ & 101.93 & A. soldadoensis & $300-355$ & 3.42 & -2.19 & 3 \\
\hline $6 \mathrm{H}-5,70-72$ & 53.20 & A. bullbrooki & $300-355$ & 3.18 & -0.57 & 3 & $11 \mathrm{H}-6,85-87$ & 102.35 & A. soldadoensis & $300-355$ & 3.29 & -2.12 & 2 \\
\hline $6 \mathrm{H}-6,70-72$ & 54.70 & A. bullbrooki & $300-355$ & 3.14 & -0.58 & 3 & $11 \mathrm{H}-\mathrm{CC}, 9-12 \mathrm{D}$ & 102.53 & A. soldadoensis & $300-355$ & 3.24 & -2.02 & 3 \\
\hline $7 \mathrm{H}-1,118-120$ & 57.18 & A. bullbrooki & $300-355$ & 3.26 & -0.69 & 3 & $11 \mathrm{H}-\mathrm{CC}, 9-12 \mathrm{D}$ & 102.53 & A. soldadoensis & $300-355$ & 3.34 & -2.05 & 2 \\
\hline $7 \mathrm{H}-2,78-80$ & 58.28 & A. bullbrooki & $300-355$ & 3.08 & -0.67 & 3 & $11 \mathrm{H}-\mathrm{CC}, 9-12$ & 102.53 & A. soldadoensis & $300-355$ & 3.24 & -2.05 & 2 \\
\hline $7 \mathrm{H}-3,69-71$ & 59.69 & A. bullbrooki & $300-355$ & 3.10 & -0.77 & 3 & $12 \mathrm{H}-1,0-2$ & 103.50 & A. soldadoensis & $300-355$ & 2.66 & -1.93 & 2 \\
\hline $7 \mathrm{H}-4,66-68$ & 61.18 & A. bullbrooki & $300-355$ & 3.15 & -0.68 & 3 & $12 \mathrm{H}-1,10-12$ & 103.60 & A. soldadoensis & $300-355$ & 1.29 & -1.94 & 2 \\
\hline $7 \mathrm{H}-4,66-68 \mathrm{D}$ & 61.18 & A. bullbrooki & $300-355$ & 3.03 & -0.69 & 3 & $12 \mathrm{H}-1,20-22$ & 103.70 & A. soldadoensis & $300-355$ & 3.94 & -2.02 & 2 \\
\hline $7 \mathrm{H}-5,70-72$ & 62.70 & A. bullbrooki & $300-355$ & 3.08 & -0.88 & 3 & $12 \mathrm{H}-1,30-32$ & 103.80 & A. soldadoensis & $300-355$ & 3.24 & -1.93 & 2 \\
\hline $7 \mathrm{H}-6,76-78$ & 63.76 & A. bullbrooki & $300-355$ & 2.70 & -0.85 & 3 & $12 \mathrm{H}-1,40-42$ & 103.90 & A. soldadoensis & $300-355$ & 3.90 & -2.09 & 2 \\
\hline $8 \mathrm{H}-1,89-91$ & 66.39 & A. bullbrooki & $300-355$ & 3.20 & -1.03 & 3 & $12 \mathrm{H}-1,40-42 \mathrm{D}$ & 103.90 & A. soldadoensis & $300-355$ & 3.98 & -2.07 & 2 \\
\hline $8 \mathrm{H}-2,70-72$ & 67.70 & A. bullbrooki & $300-355$ & 3.41 & -1.10 & 3 & $12 \mathrm{H}-1,70-72 \mathrm{D}$ & 104.20 & A. soldadoensis & $300-355$ & 4.10 & -2.08 & 3 \\
\hline $8 \mathrm{H}-3,70-72$ & 69.20 & A. bullbrooki & $300-355$ & 3.19 & -1.17 & 3 & $12 \mathrm{H}-1,70-72 \mathrm{D}$ & 104.20 & A. soldadoensis & $300-355$ & 4.18 & -2.03 & 3 \\
\hline $8 \mathrm{H}-4,67-69$ & 70.67 & A. bullbrooki & $300-355$ & 3.24 & -0.96 & 3 & $12 \mathrm{H}-1,70-72$ & 104.20 & A. soldadoensis & $300-355$ & 4.11 & -2.00 & 2 \\
\hline $8 \mathrm{H}-5,70-72$ & 72.20 & A. bullbrooki & $300-355$ & 2.95 & -1.28 & 3 & $12 \mathrm{H}-1,120-125$ & 104.70 & A. soldadoensis & $300-355$ & 4.32 & -2.02 & 2 \\
\hline $8 \mathrm{H}-5,70-72 \mathrm{D}$ & 72.20 & A. bullbrooki & $300-355$ & 2.80 & -1.28 & 3 & $12 \mathrm{H}-2,20-22$ & 105.20 & A. soldadoensis & $300-355$ & 3.91 & -1.90 & 2 \\
\hline $8 \mathrm{H}-6,70-72$ & 73.70 & A. bullbrooki & $300-355$ & 2.91 & -1.55 & 3 & $12 \mathrm{H}-2,70-72 \mathrm{D}$ & 105.70 & A. soldadoensis & $300-355$ & 4.04 & -1.88 & 3 \\
\hline $9 \mathrm{H}-1,13-15$ & 75.13 & A. bullbrooki & $300-355$ & 2.84 & -0.98 & 3 & $12 \mathrm{H}-2,70-72$ & 105.70 & A. soldadoensis & $300-355$ & 4.08 & -1.98 & 2 \\
\hline $9 \mathrm{H}-1,70-72$ & 75.70 & A. bullbrooki & $300-355$ & 2.56 & -1.22 & 3 & $12 \mathrm{H}-2,120-125$ & 106.20 & A. soldadoensis & $300-355$ & 4.07 & -1.97 & 2 \\
\hline $9 \mathrm{H}-1,70-72 \mathrm{D}$ & 75.70 & A. bullbrooki & $300-355$ & 2.95 & -1.10 & 3 & $12 \mathrm{H}-3,20-22$ & 106.70 & A. soldadoensis & $300-355$ & 4.35 & -1.95 & 2 \\
\hline $9 \mathrm{H}-1,120-125$ & 76.20 & A. bullbrooki & $300-355$ & 2.90 & -1.11 & 3 & $12 \mathrm{H}-3,70-72$ & 107.20 & A. soldadoensis & $300-355$ & 4.47 & -1.84 & 2 \\
\hline $9 \mathrm{H}-2,20-22$ & 76.70 & A. bullbrooki & $300-355$ & 2.92 & -1.01 & 3 & $12 \mathrm{H}-3,120-125$ & 107.70 & A. soldadoensis & $300-355$ & 4.62 & -2.01 & 2 \\
\hline $9 \mathrm{H}-2,70-72$ & 77.20 & A. bullbrooki & $300-355$ & 2.77 & -1.16 & 3 & $12 \mathrm{H}-4,70-72$ & 108.70 & A. soldadoensis & $300-355$ & 4.29 & -1.74 & 3 \\
\hline $9 \mathrm{H}-2,120-122$ & 77.70 & A. bullbrooki & $300-355$ & 2.92 & -1.23 & 3 & $12 \mathrm{H}-5,20-22$ & 109.70 & A. soldadoensis & $300-355$ & 4.19 & -1.82 & 3 \\
\hline $9 \mathrm{H}-3,20-22$ & 78.20 & A. bullbrooki & $300-355$ & 3.05 & -1.16 & 3 & & & & & & & \\
\hline $9 \mathrm{H}-3,70-72$ & 78.70 & A. bullbrooki & $300-355$ & 3.08 & -1.47 & 3 & $12 \mathrm{H}-3,70-72$ & 107.20 & A. mckannai & $300-355$ & 4.43 & -1.89 & 2 \\
\hline $9 \mathrm{H}-3,120-125$ & 79.20 & A. bullbrooki & $300-355$ & 2.89 & -1.46 & 3 & $12 \mathrm{H}-3,120-125$ & 107.70 & A. mckannai & $300-355$ & 4.52 & -2.06 & 2 \\
\hline $9 \mathrm{H}-4,10-12$ & 79.60 & A. bullbrooki & $300-355$ & 2.56 & -1.16 & 3 & $12 \mathrm{H}-4,70-72$ & 108.70 & A. mckannai & $300-355$ & 4.26 & -1.86 & 2 \\
\hline \multirow[t]{2}{*}{$9 \mathrm{H}-4,70-72$} & 80.20 & A. bullbrooki & $300-355$ & 3.04 & -1.36 & 3 & $12 \mathrm{H}-4,120-125$ & 109.20 & A. mckannai & $300-355$ & 4.43 & -2.04 & 2 \\
\hline & & & & & & & $12 \mathrm{H}-5,20-22$ & 109.70 & A. mckannai & $300-355$ & 4.28 & -1.83 & 2 \\
\hline $9 \mathrm{H}-3,120-125$ & 79.20 & A. soldadoensis & $300-355$ & 3.19 & -1.16 & 3 & $12 \mathrm{H}-5,70-72$ & 110.20 & A. mckannai & $300-355$ & 4.40 & -1.86 & 2 \\
\hline $9 \mathrm{H}-4,10-12$ & 79.60 & A. soldadoensis & $300-355$ & 1.95 & -1.01 & 3 & $12 \mathrm{H}-5,120-125$ & 110.70 & A. mckannai & $300-355$ & 4.31 & -1.73 & 3 \\
\hline $9 \mathrm{H}-4,70-72$ & 80.20 & A. soldadoensis & $300-355$ & 2.63 & -1.44 & 3 & $12 \mathrm{H}-6,20-22$ & 111.20 & A. mckannai & $300-355$ & 4.51 & -1.80 & 2 \\
\hline $9 \mathrm{H}-4,120-125$ & 80.70 & A. soldadoensis & $300-355$ & 2.55 & -1.41 & 3 & $12 \mathrm{H}-6,70-72$ & 111.70 & A. mckannai & $300-355$ & 4.77 & -1.81 & 2 \\
\hline $9 \mathrm{H}-5,18-19$ & 81.18 & A. soldadoensis & $300-355$ & 2.88 & -1.32 & 3 & $12 \mathrm{H}-6,102-104$ & 112.02 & A. mckannai & $300-355$ & 4.63 & -1.89 & 2 \\
\hline $9 \mathrm{H}-5,70-72$ & 81.70 & A. soldadoensis & $300-355$ & 2.32 & -0.99 & 3 & $12 \mathrm{H}-6,102-104 \mathrm{D}$ & 112.02 & A. mckannai & $300-355$ & 4.42 & -1.66 & 2 \\
\hline $9 \mathrm{H}-5,70-72 \mathrm{D}$ & 81.70 & A. soldadoensis & $300-355$ & 2.40 & -1.04 & 3 & $13 \mathrm{H}-1,21-23 \mathrm{D}$ & 113.21 & A. mckannai & $300-355$ & 4.37 & -1.75 & 3 \\
\hline $9 \mathrm{H}-5,120-125$ & 82.20 & A. soldadoensis & $300-355$ & 2.16 & -1.06 & 3 & $13 \mathrm{H}-1,21-23$ & 113.21 & A. mckannai & $300-355$ & 4.43 & -1.94 & 2 \\
\hline $9 \mathrm{H}-6,6-11$ & 82.56 & A. soldadoensis & $300-355$ & 2.57 & -1.62 & 3 & $13 \mathrm{H}-1,70-72$ & 113.70 & A. mckannai & $300-355$ & 4.44 & -1.57 & 2 \\
\hline $9 \mathrm{H}-6,20-22$ & 82.70 & A. soldadoensis & $300-355$ & 2.32 & -1.23 & 3 & $13 \mathrm{H}-2,17-19 \mathrm{D}$ & 114.67 & A. mckannai & $300-355$ & 4.47 & -1.83 & 3 \\
\hline $9 \mathrm{H}-6,70-72$ & 83.20 & A. soldadoensis & $300-355$ & 2.63 & -1.79 & 3 & $13 \mathrm{H}-2,17-19$ & 114.67 & A. mckannai & $300-355$ & 4.73 & -1.84 & 2 \\
\hline $10 \mathrm{H}-1,4-6$ & 84.54 & A. soldadoensis & $300-355$ & 2.44 & -2.09 & 3 & & & & & & & \\
\hline
\end{tabular}

Notes: D after cm interval refers to duplicate analysis. Run 1 took place in June 1993; Run 2 occurred in November 1993; Run 3 took place in March 1994. All analyses are reported with respect to PDB. 
Table 2. Stable isotopic data for Hole $865 \mathrm{C}$.

\begin{tabular}{|c|c|c|c|c|c|c|}
\hline $\begin{array}{l}\text { Core, section, } \\
\text { interval }(\mathrm{cm})\end{array}$ & $\begin{array}{l}\text { Depth } \\
\text { (mbsf) }\end{array}$ & Taxon & $\begin{array}{l}\text { Size } \\
(\mu \mathrm{m})\end{array}$ & $\begin{array}{l}\delta^{13} \mathrm{C} \\
(\%)\end{array}$ & $\begin{array}{l}\delta^{18} \mathrm{O} \\
(\%)(\%)\end{array}$ & Run \\
\hline \multicolumn{7}{|c|}{ Benthic foraminifers } \\
\hline $11 \mathrm{H}-5,130-132$ & 96.10 & Cibicidoides sp. & $>125$ & 0.78 & -0.15 & 1 \\
\hline $12 \mathrm{H}-1,111-113$ & 99.41 & Cibicidoides sp. & $>125$ & 0.98 & -0.49 & $\mathrm{i}$ \\
\hline $12 \mathrm{H}-2,20-22$ & 100.00 & Cibicidoides sp. & $>125$ & 1.07 & -0.42 & 1 \\
\hline $12 \mathrm{H}-2,111-113$ & 100.91 & Cibicidoides sp. & $>125$ & 1.13 & -0.36 & 1 \\
\hline $12 \mathrm{H}-3,20-22$ & 101.50 & Cibicidoides sp. & $>125$ & 1.04 & -0.19 & 1 \\
\hline $12 \mathrm{H}-3,100-102$ & 102.30 & Cibicidoides sp. & $>125$ & 0.51 & -0.44 & 1 \\
\hline $12 \mathrm{H}-3,111-113$ & 102.41 & Cibicidoides sp. & $>125$ & 0.61 & -0.27 & 1 \\
\hline $12 \mathrm{H}-3,120-122$ & 102.50 & Cibicidoides sp. & $>125$ & 0.00 & -1.16 & 1 \\
\hline $12 \mathrm{H}-3,130-132$ & 102.60 & Cibicidoides sp. & $>125$ & 0.38 & -0.45 & 1 \\
\hline $12 \mathrm{H}-3,130-132 \mathrm{D}$ & 102.60 & Cibicidoides sp. & $>125$ & 0.23 & -0.47 & 1 \\
\hline $12 \mathrm{H}-3,140-142$ & 102.70 & Cibicidoides sp. & $>125$ & -0.76 & -2.59 & 1 \\
\hline $12 \mathrm{H}-3,140-142 \mathrm{D}$ & 102.70 & Cibicidoides sp. & $>125$ & 0.26 & -0.85 & 1 \\
\hline $12 \mathrm{H}-3,140-142 \mathrm{D}$ & 102.70 & Cibicidoides sp. & $>125$ & -0.01 & -0.49 & 3 \\
\hline $12 \mathrm{H}-4,0-2$ & 102.80 & Cibicidoides sp. & $>125$ & -0.07 & -0.76 & 1 \\
\hline $12 \mathrm{H}-4,0-2 \mathrm{D}$ & 102.80 & Cibicidoides sp. & $>125$ & 0.32 & -0.58 & 3 \\
\hline $12 \mathrm{H}-4,10-12$ & 102.90 & Cibicidoides sp. & $>125$ & 1.47 & 0.02 & 1 \\
\hline $12 \mathrm{H}-2,111-113$ & 100.91 & Lenticulina sp. & $>125$ & 0.21 & -0.19 & 1 \\
\hline $12 \mathrm{H}-3,140-142$ & 102.70 & Lenticulina sp. & $>125$ & -0.45 & -0.43 & 1 \\
\hline $12 \mathrm{H}-4,10-12$ & 102.90 & Lenticulina sp. & $>125$ & -1.69 & -1.01 & 1 \\
\hline $12 \mathrm{H}-4,30-32$ & 103.10 & Lenticulina sp. & $>125$ & -0.57 & -0.31 & 1 \\
\hline $12 \mathrm{H}-4,20-22$ & 103.00 & $N$, truempyi & $>125$ & 0.78 & -0.14 & 1 \\
\hline \multicolumn{7}{|c|}{ Planktonic foraminifers } \\
\hline $11 \mathrm{H}-6,52-54$ & 96.82 & M. subbotinae & $300-355$ & 3.78 & -1.92 & 1 \\
\hline $12 \mathrm{H}-1,10-12$ & 98.40 & M. subbotinae & $300-355$ & 3.86 & -1.97 & 1 \\
\hline $12 \mathrm{H}-1,60-62$ & 98.90 & M. subbotinae & $300-355$ & 3.80 & -2.10 & 1 \\
\hline $12 \mathrm{H}-2,70-72$ & 100.50 & M. subbotinae & $300-355$ & 3.86 & -2.03 & $i$ \\
\hline $12 \mathrm{H}-3,0-2$ & 101.30 & M. subbotinae & $300-355$ & 3.84 & -2.01 & 1 \\
\hline $12 \mathrm{H}-2,70-72$ & 100.50 & M. velascoensis & $300-355$ & 3.65 & -2.05 & 1 \\
\hline $12 \mathrm{H}-2,70-72 \mathrm{D}$ & 100.50 & M. velascoensis & $300-355$ & 3.62 & -2.09 & 1 \\
\hline $12 \mathrm{H}-3,0-2$ & 101.30 & $M$. velascoensis & $300-355$ & 3.73 & -2.17 & 1 \\
\hline $12 \mathrm{H}-3,10-12$ & 101.40 & M. velascoensis & $300-355$ & 3.58 & -2.02 & 1 \\
\hline $12 \mathrm{H}-3,30-32$ & 101.60 & M. velascoensis & $300-355$ & 3.60 & -1.91 & 1 \\
\hline $12 \mathrm{H}-3,50-52$ & 101.80 & M. velascoensis & $300-355$ & 3.68 & -2.08 & 1 \\
\hline $12 \mathrm{H}-3,60-62$ & 101.90 & M. velascoensis & $300-355$ & 3.54 & -1.89 & 1 \\
\hline $12 \mathrm{H}-3,70-72$ & 102.00 & M. velascoensis & $300-355$ & 3.53 & -2.09 & 1 \\
\hline $12 \mathrm{H}-3,80-82$ & 102.10 & M. velascoensis & $300-355$ & 3.38 & -2.04 & 1 \\
\hline $12 \mathrm{H}-3,90-92$ & 102.20 & M. velascoensis & $300-355$ & 3.33 & -1.96 & i \\
\hline $12 \mathrm{H}-3,110-112$ & 102.40 & M. velascoensis & $300-355$ & 3.16 & -1.99 & 1 \\
\hline $12 \mathrm{H}-3,120-122$ & 102.50 & M. velascoensis & $300-355$ & 3.08 & -2.03 & 1 \\
\hline $12 \mathrm{H}-3,130-132$ & 102.60 & M. velascoensis & $300-355$ & 3.00 & -2.14 & 1 \\
\hline $12 \mathrm{H}-3,140-142$ & 102.70 & M. velascoensis & $300-355$ & 4.00 & -1.88 & 1 \\
\hline $12 \mathrm{H}-3,140-142 \mathrm{D}$ & 102.70 & M. velascoensis & $300-355$ & 4.00 & -1.76 & 1 \\
\hline $12 \mathrm{H}-4,0-2$ & 102.80 & M. velascoensis & $300-355$ & 2.11 & -1.83 & 1 \\
\hline $12 \mathrm{H}-4,10-12$ & 102.90 & M. velascoensis & $300-355$ & 3.39 & -1.78 & 1 \\
\hline $12 \mathrm{H}-4,20-22$ & 103.00 & M. velascoensis & $300-355$ & 4.08 & -1.93 & 1 \\
\hline $12 \mathrm{H}-4,20-22 \mathrm{D}$ & 103.00 & M. velascoensis & $300-355$ & 3.87 & -1.87 & 1 \\
\hline $12 \mathrm{H}-4,30-32$ & 103.10 & M. velascoensis & $300-355$ & 4.00 & -1.90 & $\hat{i}$ \\
\hline
\end{tabular}

\begin{tabular}{|c|c|c|c|c|c|c|}
\hline $\begin{array}{l}\text { Core, section, } \\
\text { interval }(\mathrm{cm})\end{array}$ & $\begin{array}{l}\text { Depth } \\
\text { (mbsf) }\end{array}$ & Taxon & $\begin{array}{l}\text { Size } \\
(\mu \mathrm{m})\end{array}$ & $\begin{array}{l}\delta^{13} \mathrm{C} \\
(\%)\end{array}$ & $\begin{array}{l}\delta^{18} \mathrm{O} \\
(\%) \text { ) }\end{array}$ & Run \\
\hline $12 \mathrm{H}-4,40-42$ & 103.20 & M. velascoensis & $300-355$ & 4.28 & -1.89 & 1 \\
\hline $12 \mathrm{H}-4,50-52$ & 103.30 & M. velascoensis & $300-355$ & 4.41 & -1.94 & 1 \\
\hline $12 \mathrm{H}-4,60-62$ & 103.40 & M. velascoensis & $300-355$ & 4.36 & -1.79 & 1 \\
\hline $12 \mathrm{H}-4,70-72$ & 103.50 & M. velascoensis & $300-355$ & 4.28 & -1.83 & 1 \\
\hline $12 \mathrm{H}-4,80-82$ & 103.60 & $M$. velascoensis & $300-355$ & 4.43 & -1.94 & 1 \\
\hline $12 \mathrm{H}-4,90-92$ & 103.70 & M. velascoensis & $300-355$ & 4.51 & -1.80 & 1 \\
\hline $12 \mathrm{H}-4,110-112$ & 103.90 & M. velascoensis & $300-355$ & 4.46 & -1.77 & 1 \\
\hline $12 \mathrm{H}-4,110-112 \mathrm{D}$ & 103.90 & M. velascoensis & $300-355$ & 4.35 & -1.76 & 1 \\
\hline $12 \mathrm{H}-4,120-122$ & 104.00 & M. velascoensis & $300-355$ & 4.45 & -1.84 & 1 \\
\hline $12 \mathrm{H}-4,130-132$ & 104.10 & M. velascoensis & $300-355$ & 4.30 & -1.78 & 1 \\
\hline $12 \mathrm{H}-4,140-142$ & 104.20 & M. velascoensis & $300-355$ & 4.55 & -1.84 & 1 \\
\hline $12 \mathrm{H}-5,0-2$ & 104.30 & M. velascoensis & $300-355$ & 4.48 & -1.86 & 1 \\
\hline $12 \mathrm{H}-5,70-72$ & 105.00 & M. velascoensis & $300-355$ & 4.36 & -1.84 & 1 \\
\hline $12 \mathrm{H}-5,130-132$ & 105.60 & M. velascoensis & $300-355$ & 4.47 & -1.68 & 1 \\
\hline $12 \mathrm{H}-6,70-72$ & 106.50 & M. velascoensis & $300-355$ & 4.65 & -1.81 & 1 \\
\hline $11 \mathrm{H}-6,52-54$ & 96.82 & A. soldadoensis & $300-355$ & 3.32 & -2.10 & 2 \\
\hline $12 \mathrm{H}-1,10-12$ & 98.40 & A. soldadoensis & $300-355$ & 3.40 & -2.04 & 2 \\
\hline $12 \mathrm{H}-1,10-12 \mathrm{D}$ & 98.40 & A. soldadoensis & $300-355$ & 3.40 & -2.04 & 2 \\
\hline $12 \mathrm{H}-1,60-62$ & 98.90 & A. soldadoensis & $300-355$ & 3.40 & 2.12 & 2 \\
\hline $12 \mathrm{H}-2,70-72$ & 100.50 & A. soldadoensis & $300-355$ & 3.21 & -2.24 & 2 \\
\hline $12 \mathrm{H}-3,0-2$ & 101.30 & A. soldadoensis & $300-355$ & 3.30 & -2.14 & 2 \\
\hline $12 \mathrm{H}-3,10-12$ & 101.40 & A. soldadoensis & $300-355$ & 3.20 & -2.29 & 2 \\
\hline $12 \mathrm{H}-3,30-32$ & 101.60 & A. soldadoensis & $300-355$ & 3.32 & -1.94 & 2 \\
\hline $12 \mathrm{H}-3,40-42$ & 101.70 & A. soldadoensis & $300-355$ & 3.42 & -2.10 & 2 \\
\hline $12 \mathrm{H}-3,50-52$ & 101.80 & A. soldadoensis & $300-355$ & 3.39 & -1.98 & 2 \\
\hline $12 \mathrm{H}-3,60-62$ & 101.90 & A. soldadoensis & $300-355$ & 3.21 & -1.94 & 2 \\
\hline $12 \mathrm{H}-3,70-72$ & 102.00 & A. soldadoensis & $300-355$ & 3.54 & -2.27 & 2 \\
\hline $12 \mathrm{H}-3,70-72 \mathrm{D}$ & 102.00 & A. soldadoensis & $300-355$ & 3.12 & -2.05 & 2 \\
\hline $12 \mathrm{H}-3,80-82$ & 102.10 & A. soldadoensis & $300-355$ & 3.26 & -2.05 & 2 \\
\hline $12 \mathrm{H}-3,90-92$ & 102.20 & A. soldadoensis & $300-355$ & 2.99 & -1.95 & 2 \\
\hline $12 \mathrm{H}-3,140-142$ & 102.70 & A. soldadoensis & $300-355$ & 2.05 & -1.80 & 2 \\
\hline $12 \mathrm{H}-4,0-2$ & 102.80 & A. soldadoensis & $300-355$ & 1.91 & -1.90 & 2 \\
\hline $12 \mathrm{H}-4,10-12$ & 102.90 & A. soldadoensis & $300-355$ & 1.60 & -2.12 & 2 \\
\hline $12 \mathrm{H}-4,50-52$ & 103.30 & A. soldadoensis & $300-355$ & 4.23 & -2.04 & 2 \\
\hline $12 \mathrm{H}-4,60-62$ & 103.40 & A. soldadoensis & $300-355$ & 4.14 & -2.05 & 2 \\
\hline $12 \mathrm{H}-4,70-72$ & 103.50 & A. soldadoensis & $300-355$ & 3.93 & -1.86 & 2 \\
\hline $12 \mathrm{H}-4,80-82$ & 103.60 & A. soldadoensis & $300-355$ & 4.34 & -2.05 & 2 \\
\hline $12 \mathrm{H}-4,90-92$ & 103.70 & A. soldadoensis & $300-355$ & 4.32 & -2.04 & 2 \\
\hline $12 \mathrm{H}-4,110-112$ & 103.90 & A. soldadoensis & $300-355$ & 4.30 & -1.94 & 2 \\
\hline $12 \mathrm{H}-4,120-122$ & 104.00 & A. soldadoensis & $300-355$ & 4.26 & -2.01 & 2 \\
\hline $12 \mathrm{H}-4,130-132$ & 104.10 & A. soldadoensis & $300-355$ & 4.25 & -2.00 & 2 \\
\hline $12 \mathrm{H}-5,0-2$ & 104.30 & A. soldadoensis & $300-355$ & 4.26 & -1.96 & 2 \\
\hline $12 \mathrm{H}-5,70-72$ & 105.00 & A. soldadoensis & $300-355$ & 4.30 & -1.88 & 2 \\
\hline $12 \mathrm{H}-5,70-72 \mathrm{D}$ & 105.00 & A. soldadoensis & $300-355$ & 4.36 & -1.90 & 2 \\
\hline $12 \mathrm{H}-5,130-132$ & 105.60 & A. soldadoensis & $300-355$ & 4.10 & -1.92 & 2 \\
\hline $12 \mathrm{H}-6,70-72$ & 106.50 & A. soldadoensis & $300-355$ & 4.44 & -1.97 & 2 \\
\hline
\end{tabular}

Notes: D after $\mathrm{cm}$ interval refers to duplicate analysis. Run 1 took place in June 1993; Run 2 occurred in November 1993; Run 3 took place in March 1994. All analysesare reported with respect to PDB. 